\title{
Nanoscale
}

PAPER
View Article Online

View Journal / View Issue
A) Check for updates

Cite this: Nanoscale, 2018, 10, 4096

\section{The disclosure of mesoscale behaviour of a 3d-SMM monolayer on Au(111) through a multilevel approach $\uparrow$}

\author{
Guglielmo Fernandez Garcia, (DD ${ }^{\text {a,b }}$ Alessandro Lunghi, (DD ${ }^{a, c}$ Federico Totti (DD *a and \\ Roberta Sessoli (D) a
}

\begin{abstract}
Here we present a computational study of a full- and a half-monolayer of a $\mathrm{Fe}_{4}$ single molecule magnet $\left(\left[\mathrm{Fe}_{4}(\mathrm{~L})_{2}(\mathrm{dpm})_{6}\right]\right.$, where $\mathrm{H}_{3} \mathrm{~L}=$ 2-hydroxymethyl-2-phenylpropane-1,3-diol and $\mathrm{Hdpm}=$ dipivaloylmethane, $\mathrm{Fe}_{4} \mathrm{Ph}$ ) on an unreconstructed surface of $\mathrm{Au}(111)$. This has been possible through the application of an integrated approach, which allows the explicit inclusion of the packing effects in the classical dynamics to be used in a second step in periodic and non-periodic high level DFT calculations. In this way we can obtain access to mesoscale geometrical data and verify how they can influence the magnetic properties of interest of the single $\mathrm{Fe}_{4}$ molecule. The proposed approach allows to overcome the $a b$ initio state-of-the-art approaches used to study Single Molecule Magnets (SMMs), which are based on the study of one single adsorbed molecule and cannot represent effects on the scale of a monolayer. Indeed, we show here that it is possible to go beyond the computational limitations inherent to the use, for such complex systems, of accurate calculation techniques (e.g. ab initio molecular dynamics) without losing the level of accuracy necessary to gain new detailed insights, hardly reachable at the experimental level. Indeed, long-range and edge effects on the $\mathrm{Fe}_{4}$ structures and their easy axis of magnetization orientations have been evidenced as their different contributions to the overall macroscopic behavior.
\end{abstract}

Received 24th August 2017 Accepted 1st December 2017

DOI: $10.1039 / \mathrm{c} 7 \mathrm{nr} 06320 \mathrm{~b}$

rsc.li/nanoscale slow relaxation of their molecular magnetization at low temperature: hence the name single Molecule Magnets (SMMs). ${ }^{8}$ The possibility to retain their appealing magnetic properties in addition to the ability to tune them through an external stimulus once adsorbed on a surface can open the way to ultra-high density storage devices ${ }^{9,10}$ and to moleculebased quantum-computing. ${ }^{11}$ However, the conservation or the enhancement at the surface of the properties observed for the non-adsorbed SMM system is still an open issue. Indeed, the SMM-surface interaction can easily lead to the suppression of the target properties if wet approaches are used ${ }^{12,13}$ or even to the SMM fragmentation in the case of deposition occurring through sublimation. ${ }^{14-16}$

Such issues can be very relevant for the kind of SMM of interest in this work (polynuclear complexes). Indeed, even small surface induced structural variations and/or packing effects might dramatically influence the magnetic properties of interest, that, in the case of the SMM class of systems, are the exchange coupling constants $J$ and the magnetic anisotropy parameter $D$. Therefore, maintaining or improving the balance of the $J$ values, which define the total spin ground state $S$, and the $D$ values, which define the spin thermal relaxation barrier $\left(U \propto|D| S^{2}\right)$, becomes crucial upon surface absorption. 
For these reasons, with the increasing complexity of the system (e.g. shape or electronic structure for the adsorbed molecule and band structure for the surface) the study of the targeted properties becomes a challenging task, both at the experimental level and from a theoretical and computational point of view. The development of experiments and theoretical models, able to gain insights into such complex hybrid systems, is, therefore, a cutting-edge topic in this field. ${ }^{17}$

This work focuses on the computational study of the evolution of the deposition process on a metallic surface of single molecule magnets not just modeling it through a single SMM unit adsorbed on the surface but using a whole monolayer of SMM molecules, therefore, including also the packing effects on the properties of interest. The SMM studied in this work is the cluster $\left[\mathrm{Fe}_{4}(\mathrm{~L})_{2}(\mathrm{dpm})_{6}\right]^{18}$ where $\mathrm{Hdpm}$ is dipivaloylmethane and $\mathrm{H}_{3} \mathrm{~L}$ is the tripodal ligand 2-hydroxymethyl2-phenylpropane-1,3-diol. This $\mathrm{SMM}, \mathrm{Fe}_{4} \mathrm{Ph}$ hereafter, is a member of the propeller-shape $\mathrm{Fe}_{4}$ family and has been evaporated on an unrecostructured $\mathrm{Au}(111)$ surface. As shown by Accorsi et al. in the crystal phase these molecules have an $S=$ 5 ground state due to the coupling of the central high spin $\mathrm{Fe}^{3+}$ ion $\left(\mathrm{Fe}_{\mathrm{c}}\right)$, via double oxygen-bridges, with three peripheral high spin $\mathrm{Fe}^{3+}$ ions $\left(\mathrm{Fe}_{i}\right.$ or $\mathrm{Fe}_{1}, \mathrm{Fe}_{2}$, and $\mathrm{Fe}_{3}$, see Fig. 1).

Some fundamental and yet missing pieces of the understanding of the $\mathrm{Fe}_{4} \mathrm{Ph} @ \mathrm{Au}(111)$ system are the microscopic organization of molecules on the substrate. Although STM images with molecular resolution of $2 \mathrm{D}$ arrays have been recorded, ${ }^{19,20}$ the orientation of individual molecules (in the packing), how they interact with each other and how this interaction affects their magnetic properties are still unknown. These three aspects critically influence the properties of the final macroscopic devices, so their understanding and control is of topical interest.

From a computational perspective, both static ${ }^{7}$ (e.g. geometry optimization) and dynamical methods ${ }^{21}$ (e.g. ab initio molecular dynamics - AIMD) have been employed to study this class of adsorbed systems. The former approaches offer the chance to obtain important information within limited com-

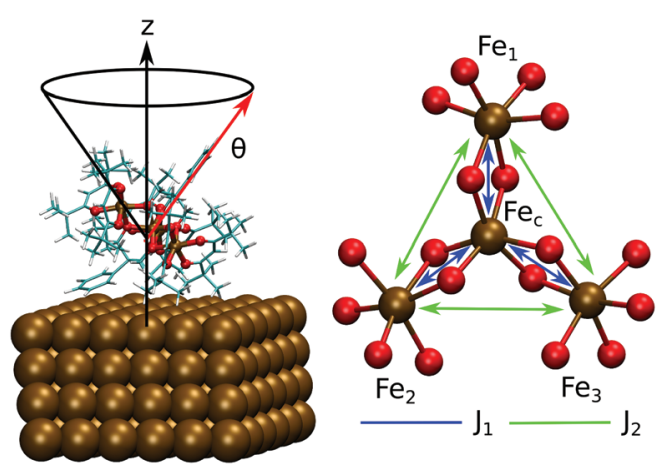

Fig. 1 Schematic representation of the $\mathrm{Fe}_{4} \mathrm{Ph}$ molecule: on the left, geometry on $\mathrm{Au}(111)\left(\mathrm{Fe}_{4} \mathrm{Ph}_{\mathrm{FM}}\right.$, vide infra) with the $\theta$ angle highlighted; on the right, the metal core of the $\mathrm{Fe}_{4}$ family along with the $J_{1}$ and $J_{2}$ couplings. putational efforts. To obtain a higher level of information, a more accurate exploration of the Potential Energy Surface (PES), to identify its multiple energy minima, is mandatory. In this framework, molecular dynamics methods become extremely valuable, as recently reported. The study of the SMM selfassembling also requires severe extensions of both length- and time-scales of simulation, up to several $\mathrm{nm}$ and $\mathrm{ns}$, respectively. To overcome inherent computational limitations of first-principles methods, we exploited a previously presented and validated multi-scale molecular dynamics methodology, ${ }^{22}$ where periodic Density Functional Theory (pDFT) is coupled to a force field (FF) based Molecular Mechanics (MM). The computational protocol is reviewed in the Methods section and in the ESI. $\dagger$ In such a framework, we addressed packing and long-range interaction effects on $\mathrm{Fe}_{4} \mathrm{Ph} @ \mathrm{Au}$ by mimicking two in silico experimental setups: for the former, a simulation cell was chosen containing a number of molecules equal to the experimental surface density (full monolayer, FM) for a total of 72 molecules, while for the latter a simulation cell with half of the molecules (half monolayer, HM) was used. For each system we analyzed both large-scale aggregation dynamics and its effect upon the internal structure of a single molecule by $\mathrm{MM} /(\mathrm{p}) \mathrm{DFT}$. Finally, to comprehensively characterize the $\mathrm{Fe}_{4} \mathrm{Ph} @ \mathrm{Au}$ system we also calculated the magnetic properties for each significant conformation highlighted by pDFT/DFT. Through our approach, it was possible to overcome the computational limitations of more accurate calculation techniques (e.g. AIMD), without losing the desired information. Even more important, it was possible to gain new insights at a higher level of detail, hardly reachable at the experimental level. Indeed, the different contributions of the long-range and edge effects on the $\mathrm{Fe}_{4}$ structure and their orientations to the overall macroscopic behavior have been evidenced. To our knowledge, this is the first time that insights at the mesoscopic level have been obtained for a structurally complex SMM, such as $\mathrm{Fe}_{4} \mathrm{Ph}$.

\section{Methods and computational details}

The flow-chart of the adopted computational protocol is sketched in Fig. 2 and it represents the extension of the one previously developed and validated on the single $\mathrm{Fe}_{4} \mathrm{Ph}$ molecule adsorbed on $\mathrm{Au}(111){ }^{22}$ The main computational details are shown below.

\subsection{Force field calculations}

The Hessian obtained at the pDFT level (vide infra) for the $\mathrm{Fe}_{4}$ system was used to derive a full set of parameters for the description of the metal core via a classical potential (Force Field - FF). The reference FF is $\mathrm{GAFF}^{23,24}$ where the bonding between the metal and the ligands is described by a simple harmonic function, while the non-bonded interactions are described by a 6-12 Lennard-Jones potential and a Coulomb term. A new modified GAFF was validated through a full 


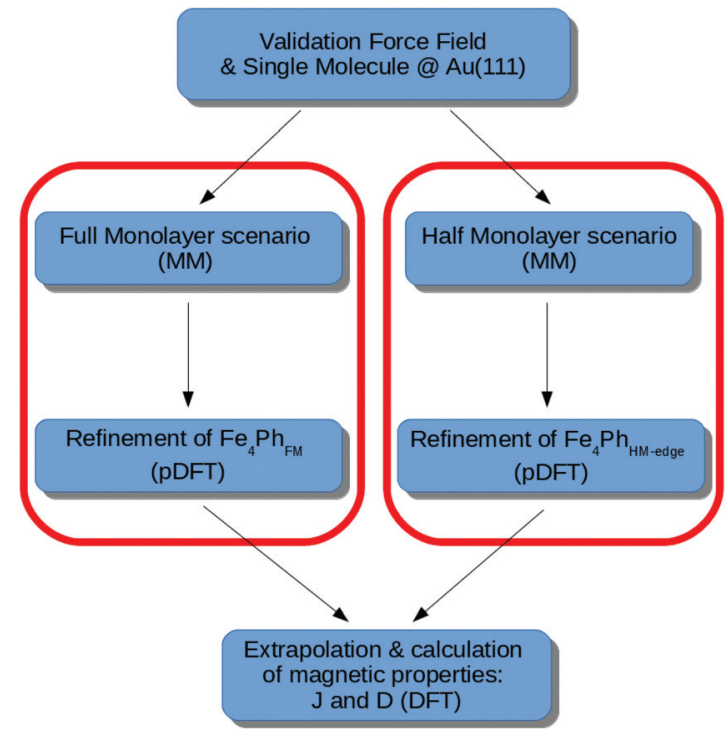

Fig. 2 Flowchart of the approach proposed in the paper, for both the Full Monolayer (FM) and Half Monolayer (HM) scenario.

benchmark testing procedure on model systems. Among them, since the inter-cluster interactions are fundamental for an accurate description of the packing dynamics, we chose to reproduce the crystal packing of $\mathrm{Fe}_{4} \mathrm{Me}$ which is isostructural to $\mathrm{Fe}_{4} \mathrm{Ph}$. The accurate results achieved indicate that standard GAFF non-bonded terms are suited for reproducing inter-Fe ${ }_{4}$ interactions. This was not true for $\mathrm{Au}-\mathrm{H}_{\text {aromatic }}$ and $\mathrm{Au}-\mathrm{C}_{\text {aromatic }}$ which had to be mimicked by an ad hoc Morse term, instead. More details are reported elsewhere. ${ }^{22}$

Both the simulation cells for FM and HM scenarios are hexagonal and their dimensions are $138 \times 134 \times 80 \AA$ A. The $\mathrm{Au}(111)$ surface is represented by six layers with the bottom one held fixed to mimic the bulk. The number of the $\mathrm{Fe}_{4} \mathrm{Ph}$ present in the cell is 72 (33120 total atoms including the surface) and 36 (24336 total atoms) for FM and HM, respectively. All $\mathrm{MD} / \mathrm{MM}$ calculations are performed with the LAMMPS package ${ }^{25}$ with Ewald summation $\left(10^{-6}\right.$ accuracy in forces), time step of $1 \mathrm{fs}$, and cutoff of pairwise interactions of $20 \AA$ with tail corrections. The equation of motion was integrated with a standard velocity-Verlet integrator. Before all simulation runs, we performed geometry optimization with the Hessian-free truncated Newton algorithm and a thermalization run of $1 \mathrm{~ns}$, with temperature rescaling, up to $300 \mathrm{~K}$. All the MD runs lasted $10 \mathrm{~ns}$ in the microcanonical ensemble ( $N V E$, constant number of particles, $N$, volume, $V$, and energy, $E$ ).

\subsection{Ab initio cell set up from MD dynamics cells}

To access the electronic information (pDFT) of the MM/MD structures containing thousands of atoms and, at the same time, saving the packing effects, we had to reduce the cell where only one $\mathrm{Fe}_{4} \mathrm{Ph}$ is present. To do so, we cooled the MM structure down to $200 \mathrm{~K}$ with a $0.5 \mathrm{~ns}$ run and through a tai- lored cut we extracted the pDFT cell from the MM/MD one. For the FM system, this resulted in a hexagonal $17 \times 17 \times 60 \AA$ cell (388 atoms of which $144 \mathrm{Au}$ ), while for the HM system a $20 \times 23 \times 60 \AA$ cubic cell has been used. Such new cells were also refined by a thermal annealing procedure at the pDFT level lowering the temperature to $0 \mathrm{~K}$. It is important to emphasize that this structure is not representative of the full ensemble explored by the simulation, but just a single sampling. However, previous studies ${ }^{21,22}$ already explored the reliability of this procedure and its limits, so, based on these grounds, we employed it as an assay to gain partial but reliable insights into the packing effects on the single molecule properties.

\subsection{Ab initio calculations}

$\mathrm{CP}_{2} \mathrm{~K}^{26}$ and $\mathrm{ORCA}^{27}$ packages were employed for the DFT description. For all the calculations involving the metal surface the CP2K code was used with the meta-GGA TPSS functional, ${ }^{28} \mathrm{rVV} 10$ corrections for dispersion forces ${ }^{29}$ and periodic boundary conditions (PBC). The CP2K code uses a GPW approach (hybrid Gaussian - Plane Wave) in which the electronic density obtained by the Kohn-Sham orbitals in a GTO basis set is mapped to an auxiliary plane wave basis set. The molecular optimized DZVP-MOLOPT-SR-GTH basis set was used along with a cutoff of 450 Ry for the plane wave expansion. To account for the metallic character of the surface, a smearing of the occupation numbers of the molecular orbitals with a Fermi-Dirac distribution with a temperature of $1500 \mathrm{~K}$ was considered. All the simulation cells present four layers of gold with the bottom layer kept fixed to mimic the bulk and only one $\mathrm{Fe}_{4} \mathrm{Ph}$ molecule in it. Their dimensions and symmetry are chosen depending on the MM/MD results obtained for the FM and HM scenarios as discussed in details in the following sections.

\subsection{Calculation of magnetic properties}

The isotropic exchange coupling in the $\mathrm{Fe}_{4}$ family can be described by the spin Hamiltonian:

$$
H_{\text {isotropic }}=\sum_{i}^{3} J_{\mathrm{c} i} \hat{s}_{\mathrm{c}} \cdot \hat{s}_{i}+J_{2}\left(\sum_{i<j}^{3} \hat{s}_{i} \cdot \hat{s}_{j}\right)
$$

$J_{\text {ci }}$ accounts for the coupling of the central $\mathrm{Fe}_{\mathrm{c}}$ with the $\mathrm{Fe}_{i}$ (peripheral ones) while from now on, we note $J_{1}$ the average of the three values. $J_{2}$ accounts for the coupling between the peripheral ions. For the latter only the average value is calculated. All the exchange couplings were computed only for the extrapolated conformers: calculations were done on the thermal-annealed pDFT geometries for the $\mathrm{Fe}_{4} \mathrm{Ph} @ \mathrm{Au}(111)$ system but erasing the gold atoms. Such a choice originates from previous evidence that the electronic effects induced by the $\mathrm{Au}(111)$ on $\mathrm{Fe}_{4} \mathrm{Ph}^{30}$ and $\mathrm{Fe}_{4} \mathrm{C}_{5}{ }^{21}$ are negligible at difference in the geometrical deformations induced by the adsorption process. The isotropic exchange coupling constants were computed within the broken symmetry ${ }^{31,32}$ framework with the PBE0 + rVV10 functional. ${ }^{33}$ The calculations of anisotropic 
tensors for the ground state $S=5, \mathbf{D}_{\mathbf{S}=5}$, were performed within the Giant Spin Approximation (GSA):

$$
\begin{aligned}
& H_{\text {aniso }}=\sum_{i}^{4} \hat{s}_{i} \cdot \mathbf{D}_{\mathrm{i}} \cdot \hat{s}_{i} \\
& H_{\text {aniso }}^{\text {GSA }}=\mathbf{S} \cdot \mathbf{D}_{\mathbf{S}=\mathbf{5}} \cdot \mathbf{S}
\end{aligned}
$$

where $\mathbf{D}_{\mathbf{i}}$ is the single ion anisotropic tensor. The anisotropic intensity and its distortion from the perfect axial symmetry are conventionally described with the axial $(D)$ and rhombic $(E)$ parameters, where $D$ eigenvalues are chosen as $0<|E / D|<1 / 3$. Diamagnetic substitution of $\mathrm{Fe}^{3+}$ ions with $\mathrm{Ga}^{3+}$ ions was used to calculate the single ion anisotropic terms and the $g$ tensors. For the calculations of anisotropic tensors (vide infra) we used the ORCA code ${ }^{27}$ with PBE functional, ${ }^{34}$ the def2-TZVP/J basis set for iron and oxygen atoms and def2-SVP for all the others. More details about the validation of the computational protocol for the calculation of the anisotropic tensors can be found elsewhere. ${ }^{35}$

\section{Results and discussion}

\subsection{Full monolayer (FM) scenario - MM structural characterization}

The scenario, in which the packing and long-range effects are maximized, is the one where the highest density of $\mathrm{Fe}_{4} \mathrm{Ph}$ is obtained. Relying on the fact that the substrate-molecule interaction has been previously elucidated by us, ${ }^{22}$ to understand the effects of the close packing arrangement on $\mathrm{Fe}_{4} \mathrm{Ph}$ 's structure, conformations and, consequently, on its magnetic properties, we have chosen to mimic the scenario in which the highest coverage density value of $\mathrm{Fe}_{4} \mathrm{Ph}$ was derived by experimental STM images ${ }^{20}$ (see Fig. 3d). Fig. 3c shows a snapshot after the geometry optimization and at the end of the $10 \mathrm{~ns}$ (see the $\mathrm{ESI} \dagger$ for the initial snapshot): the $\mathrm{Fe}_{4} \mathrm{Ph}$ monolayer exhibits extensive and stable bidimensional hexagonal-like domains. In detail, once optimized, the system readily evolves in the first ns towards an almost regular compact pattern which is retained over the complete trajectory. Indeed, being that the $\mathrm{Fe}_{4} \mathrm{Ph}$ molecules are quasi-spherical and not charged, the inter-molecular interactions are mainly driven by van der Waals interactions. In this case, the hexagonal packing is the most favorable one since it maximizes the number of contacts and, consequently, the density of $\mathrm{Fe}_{4} \mathrm{Ph}$ on gold. The calculated average distance between the $\mathrm{Fe}_{\mathrm{c}}$ (mass center) of different molecules along all the trajectory and averaged on all the possible couples shows to be in very good agreement with the average one measured between the spherical objects present in the STM images and associated with the $\mathrm{Fe}_{4} \mathrm{Ph}$ molecules. ${ }^{20}$ Moreover, in order to verify how much regular the $\mathrm{Fe}_{4} \mathrm{Ph}$ packing symmetry is, we have calculated the $g(r)$ (radial distribution function) between the $\mathrm{Fe}_{\mathrm{c}}$ of different molecules. The $g(r)$ plot, as shown in Fig. $3 \mathrm{~b}$, presents regular patterns with several maxima and minima spaced by almost zero values. The latter indicates the absence of important diffusion processes in

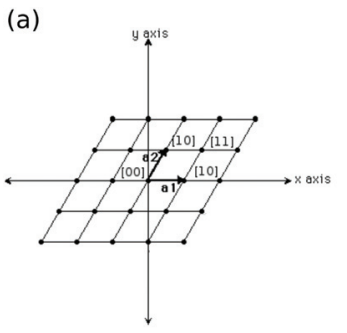

(c)

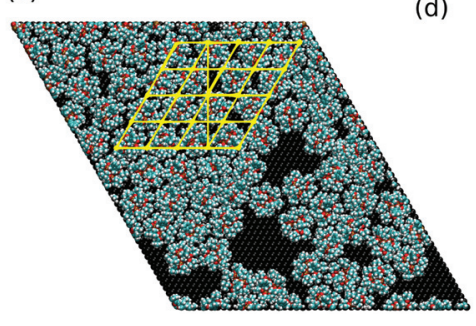

Fig. 3 (a) Bidimensional hexagonal Miller notation; (b) radial distribution function $(g(r))$ of the intermolecular distance along with the interpretation of the first peaks in terms of Miller indices; (c) final snapshot of the MM dynamic run for the FM scenario along with a hexagonal lattice (yellow lines); (d) experimental STM images reported by Malavolti et al. ${ }^{20}$

the trajectory and a high level of order in the monolayer. Similar to the solid state case, we can map each peak in Fig. $3 \mathrm{~b}$ through 2D Miller indices (Fig. 3a). In such a framework, the first sharp peak would correspond to the [10] plane and, consequently, to an estimate of the $\mathrm{Fe}_{4} \mathrm{Ph}$ monolayer lattice constant. The other peaks, whose slightly broader shapes reveal a thermal contribution, present a regular recurrence, which maps a hexagonal lattice. As already observed in the single $\mathrm{Fe}_{4} \mathrm{Ph}$ molecule, no particular contact has been found between the $\mathrm{Au}(111)$ and $\mathrm{Fe}_{4} \mathrm{Ph}$ monolayer lattices, ruling out any surface imprinting effects on the symmetry of the $\mathrm{Fe}_{4} \mathrm{Ph}$ monolayer.

Such a result is of extreme importance since the presence of a sort of "crystallographic" symmetry represents a prerequisite for $2 \mathrm{D}$ engineering and the exploitation of system properties of relevance for technological applications. ${ }^{36}$ The other fundamental aspect resides in the eventual correlation between the supramolecular order and the magnetic properties. ${ }^{37}$ With the aim of exploring this aspect, we have studied the evolution of the angle $\theta$, defined as the direction of the easy-axis of magnetization with the normal to the surface (see Fig. 1), for each of the adsorbed $\mathrm{Fe}_{4} \mathrm{Ph}$ molecules. As already shown in previous studies, ${ }^{9}$ the direction of the magnetization vector in the $\mathrm{Fe}_{4}$ family is approximately perpendicular to the plane formed by the four iron atoms. Two distributions of $\theta$ were calculated (see Fig. 4). Distribution (A) is the average of the $\theta$ distributions calculated for each single $\mathrm{Fe}_{4} \mathrm{Ph}$ in the whole trajectory. Instead (B) is the $\theta$ distribution averaged at each time-step for all the 72 $\mathrm{Fe}_{4} \mathrm{Ph}$ molecules. The two quantities give different insights into the time evolution of the monolayer: (A) is an estimation of the possible range of $\theta$ values spanned during the dynamics by each single $\mathrm{Fe}_{4} \mathrm{Ph}$ (i.e. single molecule properties), but aver- 


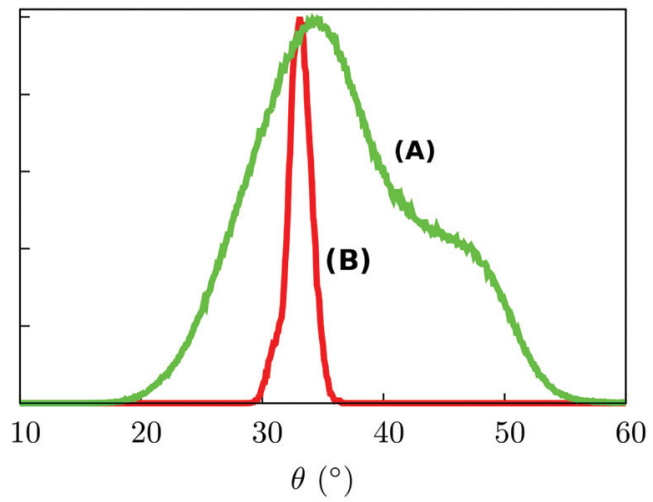

Fig. 4 (A) Represents the distribution curve of the $\theta$ value; (B) represents the distribution curve of the $\theta$ mean value. Both curves refer to the MM dynamics of the FM scenario. The probabilities are expressed in arbitrary units.

aged on the ensemble of molecules; (B) is the distribution of the mean value (i.e. average ensemble properties), where the weight of the single molecule fluctuations at a given time-step is reduced by the procedure. For such a reason, (B) distribution can be directly compared with the experimental values obtained by those experimental techniques sensitive to the average orientation of the molecule on the surface as, for example, an X-ray magnetic circular dichroism (XMCD) experiment. In such a framework, (B) is in good agreement with previous calculations and experimental findings. ${ }^{20}$ It is important to note that (B) distribution computed for the FM scenario is slightly different from the one computed for the single $\mathrm{Fe}_{4} \mathrm{Ph}$ molecule $\left(\theta=30^{\circ}\right)$, hinting an active packing effect. In contrast, (A) distribution seems to be a superposition of two broad bands centered at two different $\theta$ values: $34^{\circ}$ and $44^{\circ}$. This means that each single molecule can span a wider range of $\theta$ values than suggested by the (B) distribution and that a larger number of $\mathrm{Fe}_{4} \mathrm{Ph}$ molecules span around $\theta=34^{\circ}$ than $\theta=44^{\circ}$. However, on the basis of the results obtained for the single molecule in a previous work ${ }^{22}$ the actual $\theta$ range values can be considered the most likely to be spanned by $\mathrm{Fe}_{4} \mathrm{Ph}$ and, therefore, other significant distributions can be excluded.

With the aim to identify the two different sub-ensembles of $\mathrm{Fe}_{4} \mathrm{Ph}$ molecules, we explored the distribution of each single molecule without making the average on the whole trajectory that leads to (A). In Fig. 5a the distribution of the maximum $\theta$ value found for each single molecule distribution is shown. It is possible to observe two main distributions centred at $34^{\circ}$ and $44^{\circ}$, respectively. The latter is pretty wide while the former is narrower and accounts for a much larger number of $\theta$ values. However, such an approach does not allow the demonstration of more complex behaviors, i.e. the presence of distributions with two or more maxima. For this reason, we divided the distributions of $\theta$ in three families and we mapped each one on the last snapshot of the trajectory (in Fig. 5b the distributions of three representative molecules are reported while in Fig. $5 \mathrm{c}$ the last snapshot is presented): one distribution is peaked at $44^{\circ}$ (color green); one distribution peaked at $34^{\circ}$ (color blue); one wide distribution spanning the two other $\mathrm{Fe}_{4} \mathrm{Ph}$ sub-ensembles (color red). Such an analysis revealed that distribution (A) is not a superposition of only two distributions, but a third sub-ensemble of molecules is present. The mapping shows a detailed degree of disorder that was not evident from the mean values calculated for (A) and (B) distributions. From such an analysis, it has been possible to evidence that when a hexagonal monolayer-like packing is present (Fig. 5b), a single color is dominant (cyan colored spheres); however, in the presence of lower molecule density a more disordered pattern (different colors) is observed. Interestingly, this mapping procedure hints the presence of subtle border effects, i.e. where the density of the packing is lower. In detail, $30 \%$ of the population shows this distribution. We also note the presence of broad distribution for $9 \%$ of the total population, referring to molecules experiencing a bulklike environment that occasionally visit the conformation with $\theta$ at $44^{\circ}$. On the basis of this analysis, we can affirm that the averaged values of $\theta$ are close but different from the ones (a)

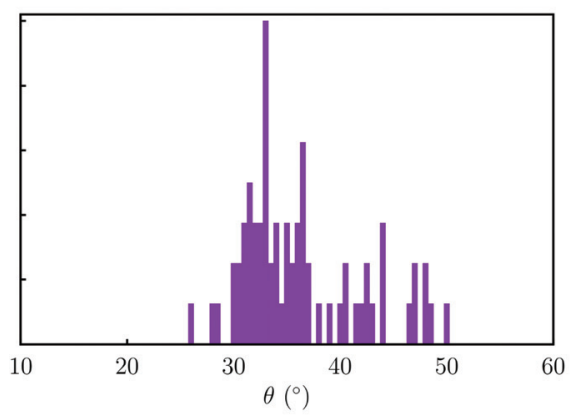

(b)

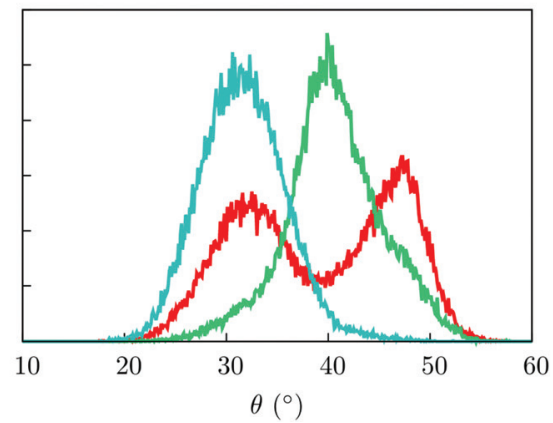

(c)

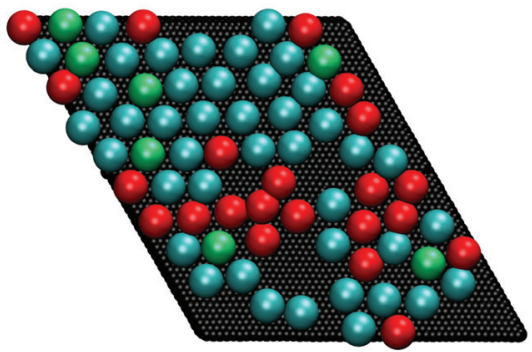

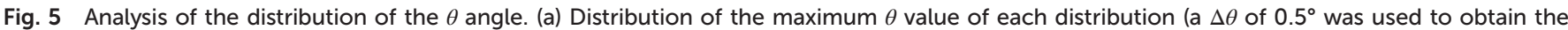

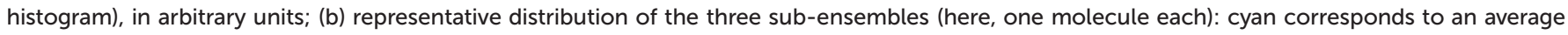

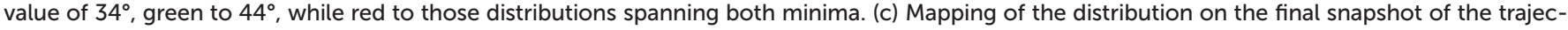
tory (each molecule is represented as a sphere) with the same color code used in (b). 
found for the isolated molecule and that they are also affected by the density of the $\mathrm{Fe}_{4} \mathrm{Ph}$ molecules.

\subsection{FM scenario - pDFT structural refining and magnetic characterization}

To obtain information on the electronic structure it is necessary to refine the MM results at a pDFT level as already presented. ${ }^{22}$ Since we are interested, first of all, to gain insights into the fine structural effects induced by the monolayer packing, we chose to perform thermal annealing on a single $\mathrm{Fe}_{4} \mathrm{Ph}$ molecule experiencing a hexagonal packing, i.e. corresponding to the $\mathrm{Fe}_{4} \mathrm{Ph}$ molecules colored with cyan (highest density). To do so, we tailored the pDFT cell on the basis of the hexagonal packing observed in the MM simulation cell as explained in Methods and computational details. The interaction with the neighboring $\mathrm{Fe}_{4} \mathrm{Ph}$ molecules is assured by periodic boundary conditions. The final geometry obtained by the thermal annealing refinement (called $\mathrm{Fe}_{4} \mathrm{Ph}_{\mathrm{FM}}$ ) shows close resemblance for both the metal core and ligands with the one obtained from the same computational protocol when only a single molecule adsorbed on $\mathrm{Au}(111)$ was considered (see the ESI $\dagger$ for all the important internal degrees of freedom). For example Table 1 shows the values of the improper dihedral angle $\phi$ of the phenyl ring of the ligand towards the surface. As already seen for a single molecule on $\mathrm{Au}(111)$, the $\phi$ angle is an important structural parameter to understand the interaction with the surface. It is clear that it does not vary substantially in the two cases, confirming the similarity of the observed structures. However, magnetic properties are strictly correlated the with structural parameters ${ }^{35,38}$ of the metal core such as the distance $\mathrm{Fe}_{\mathrm{c}}-\mathrm{Fe}_{\mathrm{p}}$, the angle FeÔFe and the $\gamma$-pitch angle. The metal core shows a more pronounced deviation from the pseudo- $C_{3}$ symmetry towards a $C_{2}$ one, but FeÔFe and $\gamma$-pitch angles (as reported in Table 1) show values that are in the high probability region in the distribution curve computed for the single $\mathrm{Fe}_{4} \mathrm{Ph}$ molecule. ${ }^{22}$ The packing effects can be somehow correlated to the average of the improper dihedral angles $\mathrm{C}_{\text {hfac,central }} \mathrm{O}_{\text {hfac }} \mathrm{O}_{\text {hfac }} \mathrm{Fe}, \delta$, related to the hfac ${ }^{-}$ligands (see the $\mathrm{ESI}^{\dagger}$ ): indeed, it is possible to observe a trend as $\delta$ (Exp.) $>\delta$ (single $\left.\mathrm{Fe}_{4} \mathrm{Ph}\right)>\delta\left(\mathrm{Fe}_{4} \mathrm{Ph}_{\mathrm{HM} \text {-edge }}\right)>\delta\left(\mathrm{Fe}_{4} \mathrm{Ph}_{\mathrm{FM}}\right)$, suggesting a non-innocent effect of the monolayer formation.

A larger isotropic coupling constant $J_{1}$ is observed (21.36 $\mathrm{cm}^{-1}$ vs. $16.05 \mathrm{~cm}^{-1}$ found for the single molecule on the surface) resulting in an increase of $20 \mathrm{~cm}^{-1}$ of the separation between the ground $S=5$ state and the first excited $S=4$

Table 1 Main structural parameters correlated with magnetic pro-

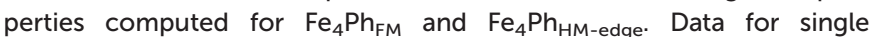
$\mathrm{Fe}_{4} \mathrm{PhaAu}$ are taken from a previous work ${ }^{22}$ (thermal annealed structure). Exp. data for the bulk reported by Accorsi et al. ${ }^{18}$

\begin{tabular}{|c|c|c|c|c|}
\hline & $<\mathrm{FeÔFe}>\left(^{\circ}\right)$ & $<\gamma$-Pitch $>\left({ }^{\circ}\right)$ & $<\mathrm{Fe}_{\mathrm{c}}-\mathrm{Fe}_{\mathrm{p}}>(\AA)$ & $\phi\left({ }^{\circ}\right)$ \\
\hline $\mathrm{Fe}_{4} \mathrm{Ph}_{\mathrm{FM}}$ & 103.7 & 68.6 & 3.12 & 15.2 \\
\hline $\mathrm{Fe}_{4} \mathrm{Ph}_{\text {HM-edge }}$ & 102.4 & 70.5 & 3.14 & 90 \\
\hline Single $\mathrm{Fe}_{4} \mathrm{Ph} @ A u$ & 102.6 & 62.2 & 3.10 & 15.7 \\
\hline Exp. & 102.2 & 68.9 & 3.07 & - \\
\hline
\end{tabular}

state. Regarding $D$ and $E / D$, only small differences with respect to the bulk have been found, as for the single $\mathrm{Fe}_{4} \mathrm{Ph} @ \mathrm{Au}(111)$ (Table 2). Moreover, as suggested by distribution (B) in Fig. 4, the narrower distribution of $\theta$ values indicates that the anisotropy axis orientations are expected to be restricted to the cone defined by the $\theta$ angle, in agreement with what was experimentally observed. ${ }^{7}$ We can conclude that the inclusion of the packing effects is likely not to introduce sensitive modifications on the core structure and, therefore, on the magnetic structure.

Having access to the $g$ tensor of the individual Fe ions, we have also calculated the dipolar contribution to the isotropic exchange interactions, i.e. the non-vanishing trace of the dipolar interaction, taking a pair of neighboring $\mathrm{Fe}_{4} \mathrm{Ph}$ as the upper limit (see the ESI $\dagger$ ). The computed $J_{\text {dip }}$ 's are of order of $10^{-7} \mathrm{~cm}^{-1}$ as expected for the long intermolecular Fe-Fe distances. The leading anisotropic part of the dipolar interaction depends on the magnetic polarization of the monolayer and its evolution during the magnetization process is known to affect resonant tunneling of the magnetization. ${ }^{39}$ The investigation of this aspect will be the subject of a future publication.

\subsection{Half monolayer (HM) scenario - MM structural characterization}

To verify whether the FM results were somehow biased by the chosen experimental high density of $\mathrm{Fe}_{4} \mathrm{Ph}$ on gold, we repeated the procedure starting from a halved number of $\mathrm{Fe}_{4} \mathrm{Ph}$ molecules on the same surface. In other words, we aimed at understanding if the system reaches an ordered state spontaneously, starting from a geometry far away from the full monolayer scenario. We have chosen the HM model because it represents a good compromise between the possibility of having a sparse guess and at the same time a significant chance to observe effective $\mathrm{Fe}_{4} \mathrm{Ph}$ interactions in a relatively short time for an MD simulation. For other concentrations as

Table 2 Isotropic exchange couplings and anisotropies computed at the pDFT and DFT level respectively. The experimental data for the J's and $D_{S=5}$ 's are taken from ref. 18 , while the single ion anisotropies from ref. 40

\begin{tabular}{lrrl}
\hline$\left(\mathrm{cm}^{-1}\right)$ & $\mathrm{Fe}_{4} \mathrm{Ph}_{\mathrm{FM}}$ & $\mathrm{Fe}_{4} \mathrm{Ph}_{\text {HM-edge }}$ & Exp. \\
\hline$J_{\mathrm{c} 1}$ & 21.05 & 22.57 & - \\
$J_{\mathrm{c} 2}$ & 23.91 & 18.26 & - \\
$J_{\mathrm{c} 3}$ & 19.13 & 23.87 & - \\
$J_{1}$ & 21.36 & 21.57 & 16.37 \\
$J_{2}$ & 0.25 & 0.03 & 0.29 \\
& & & - \\
$D_{\mathrm{c}}$ & -1.22 & -0.96 & - \\
$E / D_{\mathrm{c}}$ & 0.23 & 0.21 & 0.71 \\
$D_{1}$ & 0.35 & 0.54 & 0.11 \\
$E / D_{1}$ & 0.28 & 0.15 & 0.60 \\
$D_{2}$ & 0.58 & 0.45 & 0.17 \\
$E / D_{2}$ & 0.12 & 0.20 & 0.60 \\
$D_{3}$ & 0.66 & 0.59 & 0.17 \\
$E / D_{3}$ & 0.14 & 0.14 & -0.42 \\
$D_{S=5}$ & -0.42 & -0.41 & 0.02 \\
$E / D_{S=5}$ & 0.13 & 0.07 &
\end{tabular}


$1 / 3$ and $2 / 3$, for instance, only one of the two above conditions would be privileged with respect to the other. As evident in the snapshot reported in Fig. 6, the $\mathrm{Fe}_{4} \mathrm{Ph}$ molecules readily start to arrange themselves in the hexagonal packing as soon as they start interacting with each other. In the following, the formation of clusters with a hexagonal pattern becomes even more evident and it can be straightforwardly supposed that they can act as seeding structures for a more compact and extended surface covering. To ensure that the convergence on that ordered stucture is not biased by the starting geometry and its symmetry, trial calculations (1 ns after thermalization) were performed on a cubic cell, with a starting position in which the $\mathrm{Fe}_{4} \mathrm{Ph}$ molecules were positioned on a square 2D lattice. Also in this case we observed the same hexagonal pattern after the first ns (see the ESI $\dagger$ ).

On the basis of these results, the analysis of the hexagonal cell is consistent with the one reported for the FM case. In such a scenario, most of the molecules present a very similar structure to those examined in the FM scenario while a few $(11 \%)$ of them have explored a different minimum of the conformational space. Indeed, the latter present a $\theta$ angle equal to $90^{\circ}$. They are found in low density regions of the surface that correspond to borders of the self-assembled aggregates, suggesting the presence of domain edge effects (from now on $\mathrm{Fe}_{4} \mathrm{Ph}_{\text {HM-edge }}$ ). The possibilities of having a low percentage of $\mathrm{Fe}_{4} \mathrm{Ph}$ owing to a $\theta$ larger than $35^{\circ}$ was somehow predicted by Mannini et al.: ${ }^{7}$ indeed, the XMCD data therein presented were fitted by considering also the presence of a low percentage of $\mathrm{Fe}_{4} \mathrm{Ph}$ molecules with $\theta$ larger than $35^{\circ}$. However, it is important to point out that any other direct or indirect indication of the presence of such a conformer is not present in the literature. Notably this new $\mathrm{Fe}_{4} \mathrm{Ph}_{\mathrm{HM} \text {-edge }}$ conformer was not accessible in the single molecule $\mathrm{MD}$, since its presence is a direct consequence of the formation of the bidimensional lattice in a low density scenario. Moreover, the interaction between this conformer and its neighbor is driven by the intermolecular $\pi-\pi$ interaction between the phenyl rings, that are found lying parallel to each other and with the surface. However, since this has been observed only for a low percentage of molecules, to have a more quantitative analysis on these aspects it would be necessary to perform a molecular dynamics study on a simulation box built starting from this conformer.

\subsection{HM scenario - pDFT structural refining and magnetic characterization}

On the basis of the similarities observed between the structures of $\mathrm{Fe}_{4} \mathrm{Ph}$ molecules in $\mathrm{FM}$ and $\mathrm{HM}$ scenarios, we focused

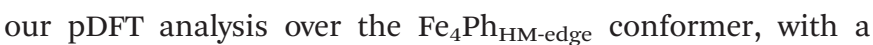
$\theta$ equal to $90^{\circ}$. We performed thermal annealing on one of these structures, following the same idea explained for $\mathrm{Fe}_{4} \mathrm{Ph}_{\mathrm{FM}}$ but, in contrast, it was not necessary to respect the geometry of the packing, since the conformer is present in low density areas (see Fig. 7). Therefore a cubic cell has been used, as explained in the Methods and computational details. The laying of the molecule with the trigonal axis parallel to the surface does not

(a)

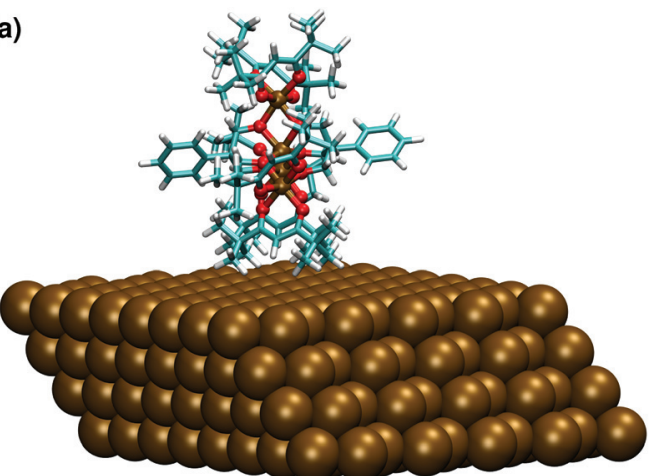

(b)

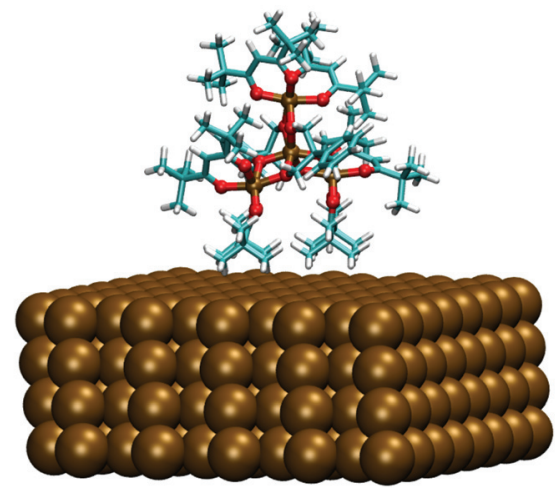

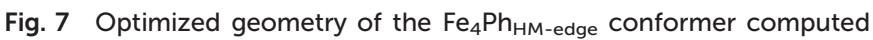
at the pDFT level: side (a) and frontal (b) view.

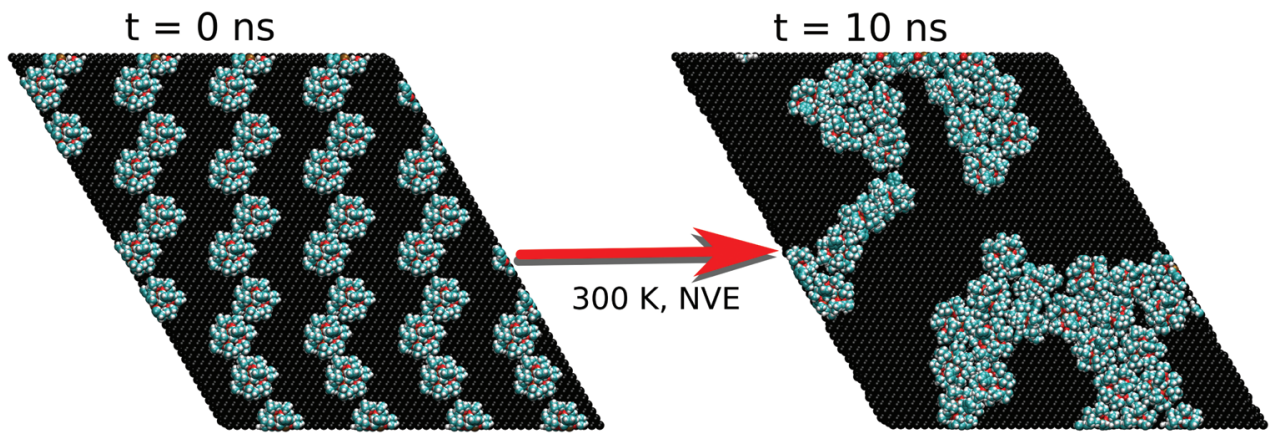

Fig. 6 First and last snapshot of the MM trajectory for the HM scenario. 
affect heavily the structural parameters (FeÔFe, $\gamma$-pitch) and the same symmetry of the metal core observed for the full monolayer $\left(\mathrm{Fe}_{4} \mathrm{Ph}_{\mathrm{FM}}\right)$ is retained. In fact, the direct contact with the surface of the hfac ${ }^{-}$ligands is reflected in an average $\delta$ dihedral angle which is comparable to the one observed for $\mathrm{Fe}_{4} \mathrm{Ph}_{\mathrm{FM}}$ (see Fig. 7 and $\mathrm{ESI} \dagger$ ). On the other hand, the metal core is shown to be quite rigid (Table 1), with only minor distortions with respect to the $\mathrm{Fe}_{4} \mathrm{Ph}_{\mathrm{FM}}$, since the major distortions are accounted for by the aforementioned more flexible $\mathrm{hfac}^{-}$ligands. Indeed, from a magnetic point of view, the isotropic couplings remain unchanged with respect to $\mathrm{Fe}_{4} \mathrm{Ph}_{\mathrm{FM}}$ and only slight differences are found for the magnetic anisotropy. Since the interaction between the phenyl ring of the tripodal ligand and the surface is weaker in this case, the $\phi$ angle results in $90^{\circ}$.

The presence of this conformer might be a "lead" to future 2D crystal engineering, in which each $\mathrm{Fe}_{4} \mathrm{Ph}$ has the terminal phenyl rings in contact with the neighbors' benzene ring, forming some sort of supramolecular chains on the surface. The possibility to form a regular and stable pattern of such conformers would represent an interesting achievement. Indeed, the disorder of the easy axis of magnetization would be significantly reduced and the orientation would be parallel to the surface. Such a situation is not common to our knowledge in the case of adsorbed SMMs. Moreover, in the case that $\mathrm{Fe}_{4} \mathrm{Ph}_{\text {HM-edge }}$ could be switched to $\mathrm{Fe}_{4} \mathrm{Ph}_{\mathrm{FM}}$ with external stimuli acting on rotovibrational degree of freedom (laser light, for instance) this would make feasible the control of the easy axis orientation of the $\mathrm{Fe}_{4} \mathrm{Ph}$ molecules in the absence of external magnetic or electric fields.

\section{Conclusions}

In this work a multi-scale molecular dynamics computational scheme has been used to study a monolayer of single molecule magnets adsorbed on a metallic surface. This novel approach made it possible to significantly extend simulation length- and time-scales unlocking the possibility to include in the model fundamental features such as the inter-molecular interactions. The model led to the corroboration of previous experimental evidence, like the formation of a $2 \mathrm{D}$ hexagonal lattice of molecules on the substrate and the overall stability of the packing. It was also able to prove, for the first time, the formation of a multitude of stable and transient conformers in the selfassembly of $\mathrm{Fe}_{4} \mathrm{Ph} @ \mathrm{Au}(111)$. These effects cannot directly be extracted from experimental analysis and their presence has so far been only predicted. Finally, a complete characterization of the spin-Hamiltonian of adsorbed $\mathrm{Fe}_{4} \mathrm{Ph}$ has revealed the effect of packing and the variability of conformers. Most importantly, these results has been analyzed from a "device" perspective, highlighting the impact of local disorder on single molecule measurements that can have an influence on the performance of the hybrid interface of molecular spintronic devices. On the other hand, the possibility to have access to several possible conformations revealed the prospect to design, for instance, new packing arrangements with different magnetic properties tunable by external stimuli. The understanding and control of the large-scale self-assembly of these systems is indeed a key factor for their technological applications and constitutes a current and future challenge in the manufacturing of molecular devices.

The level of detailed information obtained with the presented model (as the distribution of the $\theta$ angle) is something hardly achievable at the experimental level and, moreover, the MM model overcomes the intrinsic computational difficulty DFT of mimicking, on a large scale, complex systems containing transition metals.

\section{Conflicts of interest}

There are no conflicts to declare.

\section{Acknowledgements}

The authors gratefully acknowledge the support from the European Research Council (Advanced Grant No. 267746 MolNanoMaS), the European Union's Horizon2020 Research and Innovation Programme under grant agreement No. 737709 (FEMTOTERABYTE, http://www.physics.gu.se/femtoterabyte) and the MOLSPIN COST action CA15128.

\section{References}

1 A. R. Rocha, V. M. García-Suárez, S. W. Bailey, C. J. Lambert, J. Ferrer and S. Sanvito, Nat. Mater., 2005, 4, 335-339.

2 W. Wernsdorfer, Nat. Mater., 2007, 6, 174-176.

3 F. E. Kalff, M. P. Rebergen, E. Fahrenfort, J. Girovsky, R. Toskovic, J. L. Lado, J. Fernández-Rossier and A. F. Otte, Nat. Nanotechnol., 2016, 11, 926-929.

4 R. Coppage, J. M. Slocik, H. Ramezani-Dakhel, N. M. Bedford, H. Heinz, R. R. Naik and M. R. Knecht, J. Am. Chem. Soc., 2013, 135, 11048-11054.

5 M. Urdampilleta, S. Klayatskaya, M. Ruben and W. Wernsdorfer, ACS Nano, 2015, 9, 4458-4464.

6 M. Cinchetti, V. A. Dediu and L. E. Hueso, Nat. Mater., 2017, 16, 507-515.

7 M. Mannini, F. Pineider, C. Danieli, F. Totti, L. Sorace, P. Sainctavit, M.-A. Arrio, E. Otero, L. Joly, J. C. Cezar, A. Cornia and R. Sessoli, Nature, 2010, 468, 417-421.

8 D. Gatteschi, R. Sessoli and J. Villain, Molecular Nanomagnets, Oxford University Press, Oxford, 2006.

9 M. Mannini, F. Pineider, P. Sainctavit, C. Danieli, E. Otero, C. Sciancalepore, A. M. Talarico, M.-A. Arrio, A. Cornia, D. Gatteschi and R. Sessoli, Nat. Mater., 2009, 8, 194-197.

10 F. Allouche, G. Lapadula, G. Siddiqi, W. W. Lukens, O. Maury, B. Le Guennic, F. Pointillart, J. Dreiser, V. Mougel, O. Cador and C. Copéret, ACS Cent. Sci., 2017, 3(3), 244-249. 
11 M. Urdampilleta, N. V. Nguyen, J. P. Cleuziou, S. Klyatskaya, M. Ruben and W. Wernsdorfer, Int. J. Mol. Sci., 2011, 12, 6656-6667.

12 L. Rigamonti, M. Piccioli, L. Malavolti, L. Poggini, M. Mannini, F. Totti, B. Cortigiani, A. Magnani, R. Sessoli and A. Cornia, Inorg. Chem., 2013, 52, 5897-5905.

13 J. Dreiser, A. M. Ako, C. Wäckerlin, J. Heidler, C. E. Anson, A. K. Powell, C. Piamonteze, F. Nolting, S. Rusponi and H. Brune, J. Phys. Chem. C, 2015, 119, 3550-3555.

14 V. Lanzilotto, L. Malavolti, S. Ninova, I. Cimatti, L. Poggini, B. Cortigiani, M. Mannini, F. Totti, A. Cornia and R. Sessoli, Chem. Mater., 2016, 28, 7693-7702.

15 P. Erler, P. Schmitt, N. Barth, A. Irmler, S. Bouvron, T. Huhn, U. Groth, F. Pauly, L. Gragnaniello and M. Fonin, Nano Lett., 2015, 15, 4546-4552.

16 S. Rauschenbach, F. L. Stadler, E. Lunedei, N. Malinowski, S. Koltsov, G. Costantini and K. Kern, Small, 2006, 2, 540547.

17 A. Caneschi, D. Gatteschi and F. Totti, Coord. Chem. Rev., 2015, 289-290, 357-378.

18 S. Accorsi, A.-L. Barra, A. Caneschi, G. Chastanet, A. Cornia, A. C. Fabretti, D. Gatteschi, C. Mortalo, E. Olivieri, F. Parenti, P. Rosa, R. Sessoli, L. Sorace, W. Wernsdorfer and L. Zobbi, J. Am. Chem. Soc., 2006, 128, 4742-4755.

19 J. A. Burgess, L. Malavolti, V. Lanzilotto, M. Mannini, S. Yan, S. Ninova, F. Totti, S. Rolf-Pissarczyk, A. Cornia, R. Sessoli and S. Loth, Nat. Commun., 2015, 6, 8216.

20 L. Malavolti, V. Lanzilotto, S. Ninova, L. Poggini, I. Cimatti, B. Cortigiani, L. Margheriti, D. Chiappe, E. Otero, P. Sainctavit, F. Totti, A. Cornia, M. Mannini and R. Sessoli, Nano Lett., 2015, 15, 535-541.

21 A. Lunghi, M. Iannuzzi, R. Sessoli and F. Totti, J. Mater. Chem. C, 2015, 3, 7294-7304.

22 G. Fernandez Garcia, A. Lunghi, F. Totti and R. Sessoli, J. Phys. Chem. C, 2016, 120, 14774-14781.
23 J. M. Wang, R. M. Wolf, J. W. Caldwell, P. A. Kollman and D. A. Case, J. Comput. Chem., 2004, 25, 1157-1174.

24 J. Wang, W. Wang, P. A. Kollman and D. A. Case, J. Mol. Graphics Modell., 2006, 25, 247-260.

25 S. Plimpton, J. Comput. Phys., 1995, 117, 1-19.

26 J. Hutter, M. Iannuzzi, F. Schiffmann and J. VandeVondele, Wiley Interdiscip. Rev.: Comput. Mol. Sci., 2014, 4, 15-25.

27 F. Neese, Wiley Interdiscip. Rev.: Comput. Mol. Sci., 2012, 2, 73.

28 J. Tao, J. Perdew, V. Staroverov and G. Scuseria, Phys. Rev. Lett., 2003, 91, 146401.

29 R. Sabatini, T. Gorni and S. De Gironcoli, Phys. Rev. B: Condens. Matter Mater. Phys., 2013, 87, 4-7.

30 S. Ninova, V. Lanzilotto, L. Malavolti, L. Rigamonti, B. Cortigiani, M. Mannini, F. Totti and R. Sessoli, J. Mater. Chem. C, 2014, 2, 9599-9608.

31 L. Noodleman, J. Chem. Phys., 1981, 74, 5737.

32 A. Bencini and F. Totti, J. Chem. Theory Comput., 2009, 5, 144-154.

33 C. Adamo and V. Barone, J. Chem. Phys., 1999, 110, 6158.

34 J. P. Perdew, K. Burke and M. Ernzerhof, Phys. Rev. Lett., 1996, 77, 3865-3868.

35 A. Lunghi and F. Totti, J. Mater. Chem. C, 2014, 2, 8333-8343. 36 B. Moulton, J. Lu, R. Hajndl, S. Hariharan and M. J. Zaworotko, Angew. Chem., Int. Ed., 2002, 41, 28212824.

37 L. Su, W.-C. Song, J. P. Zhao and F.-C. Liu, Chem. Commun., 2016, 52, 8722-8725.

38 T. Gupta, T. Rajeshkumar and G. Rajaraman, Phys. Chem. Chem. Phys., 2014, 16, 14568.

39 L. Vergnani, A. L. Barra, P. Neugebauer, M. J. RodriguezDouton, R. Sessoli, L. Sorace, W. Wernsdorfer and A. Cornia, Chem. - Eur. J., 2012, 18, 3390-3398.

40 L. Rigamonti, A. Cornia, A. Nava, M. Perfetti, M.-E. Boulon, A.-L. Barra, X. Zhong, K. Park and R. Sessoli, Phys. Chem. Chem. Phys., 2014, 16, 17220. 